\title{
Legionella pneumophila Serogroup 8
}

National Cancer Institute

\section{Source}

National Cancer Institute. Legionella pneumophila Serogroup 8. NCI Thesaurus. Code C123505.

Subgroup 8 of Legionella pneumophila, differentiated by its antigenicity. 